\title{
NOUVELLE MÉTHODE DE DÉTECTION DE L'CESTRUS CHEZ LES BOVINS
}

\author{
J. P. SIGNORET \\ avec la collaboration technique de M. Y. de Fontaubert et J. Thibaud \\ Station de Physiologie de la Reproduction, \\ Centre de Recherches de Tours, I. N. R. A., \\ Nouzilly, 37380 Monnaie
}

\begin{abstract}
RÉSUMÉ
Un traitement hormonal comportant une série d'injections de propionate de testostérone suivi d'androgènes à effet retard produit chez la vache un niveau élevé d'activité sexuelle de type mâle. Il est possible d'utiliser une femelle ainsi préparée et équipée d'un licol marqueur pour la détection des chaleurs dans un troupeau. Trois vaches ont été utilisées dans ces conditions. Sur 49 vaches en chaleur, 47, soit 95,9 p. Ioo ont été identifiées par des marques caractéristiques au niveau du dos, tandis que les marquages de femelles non réceptives s'élevaient à 6, soit r 2 p. Ioo.

Cette méthode permet de disposer d'un animal détecteur des chaleurs, efficace, économique et d'une utilisation facile.
\end{abstract}

\section{INTRODUCTION}

La méthode de détection de l'œestrus la plus précise consiste en l'emploi d'un taureau rendu stérile par vasectomie ou déviation du pénis. Pour éviter d'avoir à effectuer des observations de longue durée, des systèmes de marquage ont été mis au point et sont proposés aux éleveurs (licols "chin ball " et " sire sine "). Ces dispositifs sont efficaces et présentent une marge d'erreurs faible; toutefois l'utilisation de mâles opérés n'est pas sans poser de problèmes. Si l'opération de vasectomie n'est pas trop onéreuse, le mâle ainsi traité continue à s'accoupler et risque de favoriser la dissémination des maladies vénériennes dans le troupeau. La déviation du pénis est une opération chirurgicale délicate qui ne peut être réalisée facilement dans un élevage. Or, l'étude des mécanismes endocriniens du comportement sexuel a montré que l'on pouvait provoquer chez la femelle l'apparition de réactions de type mâle par un traitement d'hormones androgènes : la brebis orariectomisée recevant des injections journalières de testostérone présente des éléments de comportement sexuel mâle (flairages, approches, montes) avec une fréquence comparable à ce que l'on peut observer chez le bélier (Signoret, 1972).

Nous avons donc cherché à réaliser ce comportement chez la vache par un traitement approprié et nous avons déterminé la valeur de l'animal ainsi préparé comme détecteur de l'œstrus.

Annales de Zootechnie. - 1975 . 


\section{MATÉRIEL, ETT MÉTHODES}

Le traitement que nous avons mis à l'essai comporte deux phases :

- une induction comprenant ro injections, effectuées un jour sur deux, de $200 \mathrm{mg}$ de propionate de testostérone dilué dans $4 \mathrm{ml}$ d'huile stérile ;

- un entretien consistant en une injection toutes les deux semaines de Io $\mathrm{ml}$ d'un mélange d'androgènes retard ("Interteston " Intervet).

Trois vaches de réforme de race Française Frisonne Pie Noire ont été utilisées ; l'une avait été ovariectomisée et tarie. Parmi les deux autres, non opérées, l'une était encore en lactation et l'autre tarie. Dès le début du traitement, elles ont été placées en permanence dans des lots d'une vingtaine de femelles après avoir été équipées d'un licol "chin ball ». Les enregistrements ont débuté à la fin de la phase d'induction : les marquages ont été notés chaque jour, matin et soir, tandis que la détection de l'œstrus, effectuée par présentation bi-quotidienne à un taureau vasectomisé servait de contrôle.

\section{RÉSULTATS ET DISCUSSION}

Les résultats présentés dans le tableau I montrent que la quasi-totalité des vaches en chaleur ont été marquées lors de leurs constacts avec les femelles androgénisées. Il faut remarquer cependant que l'emplacement du marquage (fig. I) est d'une grande importance pour la fiabilité de

\section{TABLEAU I}

Résultats de marquage par une vache androgénisée équipée d'un licol marqueur "Chin Ball"

\begin{tabular}{|c|c|c|c|c|c|c|}
\hline \multirow[b]{2}{*}{ Vache détectrice } & \multirow{2}{*}{$\begin{array}{l}\text { Nombre } \\
\text { de vaches } \\
\text { marquées }\end{array}$} & \multicolumn{3}{|c|}{ Nombre de vaches en chaleur } & \multicolumn{2}{|c|}{$\begin{array}{c}\text { Vaches non en chaleur } \\
\text { marquées }\end{array}$} \\
\hline & & total & marquées & p. 100 & nombre & $\begin{array}{c}\text { p. } 100 \\
\text { d'erreur } \\
\text { par exces }\end{array}$ \\
\hline \multicolumn{7}{|c|}{ Marquages en avant des hanches (Zone II) } \\
\hline $\begin{array}{c}\text { No } 934 \\
\text { intacte en lactation }\end{array}$ & 20 & 18 & 18 & 100 & 2 & 11,1 \\
\hline $\begin{array}{c}\text { No } 136 \\
\text { intacte tarie }\end{array}$ & 12 & 10 & 10 & 100 & 2 & 20 \\
\hline $\begin{array}{c}\text { No } 57 \\
\text { ovariectomisée }\end{array}$ & 21 & 21 & 19 & 90,5 & 2 & 9,5 \\
\hline Total & 53 & 49 & 47 & 95,9 & 6 & 12,2 \\
\hline \multicolumn{7}{|c|}{ Marquages sur la croupe (Zone I) } \\
\hline No 934 & 25 & 18 & 18 & 100 & 7 & 38,8 \\
\hline No 136 & 16 & 10 & 10 & 100 & 6 & 60 \\
\hline $\mathrm{N}^{\circ} \quad 57$ & 29 & 21 & 19 & 90,5 & 10 & 57,6 \\
\hline Total & 70 & 49 & 47 & 95,9 & 23 & 46,9 \\
\hline
\end{tabular}


l'identification des femelles en oestrus : de nombreuses marques situées sur la croupe (zone I) ne reflètent que des essais infructueux de chevauchement et de ce fait sont effectués aussi sur des vaches non en chaleur. Par contre, les traces localisées en avant des hanches (zone II) traduisent l'existence d'un chevauchement sur un animal immobile. L'observation des animaux a permis en effet de constater qu'elles ne se produisent qu'après un chevauchement accepté et lorsque l'animal redescend. En ne tenant compte que de ces dernières marques et sans autre observation, il est possible d'identifier les vaches en chaleur avec une marge d'erreur voisine de 5 p. roo par défaut et de Io $p$. Ioo par excès.

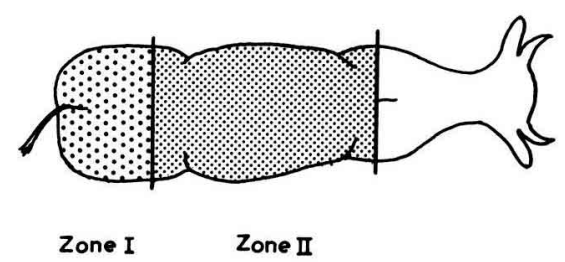

FIG. I. - Localisation des marquages par une vache traitée aux androgènes

Cette technique semble donc simple et efficace. En utilisant des vaches de réforme, on peut éviter l'introduction d'un animal étranger à l'élevage, ou les dépenses que présente l'entretien d'un jeune mâle. En outre, on élimine ainsi les risques que présente au plan sanitaire l'emploi d'un mâle vasectomisé.

Rę̧u pour publication en juillet 1974.

\title{
SUMMARY
}

\author{
NEW METHOD OF PREPARATION OF A TEASER ANIMAL, \\ FOR OESTRUS DETECTION IN CATTLE
}

Injections of $200 \mathrm{mg}$ of testosterone propionate each second day for 20 days followed by two monthly injections of long lasting androgen, induce a high level of male sexual behaviour in the cow. An androgenized female equipped with a marking harness can be success fully used as a teaser for oestrus detection in a herd : $47 / 49$ cows in heat were identified by characteristic marks on the back (95. 9 p. Ioo), 6 non receptive cows were marked ( 12 p. roo). cattle.

This method is simpler and as efficient as the use of a vasectomized bull for heat detection in

\section{RÉEÉRENCE BIBLIOGRAPHIQUE}

Signoret J. P., I972. Effet de la nature de l'hormone - cestrogène ou androgène - et du rythme d'injection sur l'apparition d'éléments de comportement sexuel mâle ou femelle chez la brebis ovariectomisée. VII' Cong. intern. Reprod. anim. Insem. artif., Munich, 1, 401-408. 